\title{
GENDER DIFFERENCE IN RISK FACTOR PROFILES IN PATIENTS REFERRED FOR CORONARY ANGIOGRAM
}

\author{
Gali Deepti, Ravi Kiran, Vanaparthi Bharathi.
}

\begin{abstract}
Background \& Objective

Coronary artery disease (CAD) is a leading cause of death of women and men worldwide. Expected burden of atherothrombotic CAD in India will surpass other regions of the world by the year 2020. It is better to control the risk factors for CAD to prevent this crisis. So, we want to study the gender difference in risk factor profiles in patients referred for coronary angiogram (CAG).

Materials $\mathcal{E}$ Methods

We did is a prospective observational study for risk factors profile in patients referred for CAG over 24 months (2013 to 2015) to the Nizam's institute of medical sciences. We collected the demographic, clinical and lab parameters including CAG finding in these patients.

Results

We analyzed the data of 3232 patients who undergone CAG during the study period. F: M::1081: 2151.706 (65.3\%) of female patients and 1312 (60.9\%) of males patients had hypertension (HTN). 496 (45.9\%) of females and 950 (44.1\%) of males had Diabetes mellitus (DM). More males were presented with ACS (Acute coronary syndrome) whereas more females were presented with CSA (chronic stable angina). 203 (18.8\%) female patients and 673 (31.3\%) had lv dysfunction. Serum creatinine and Hemoglobin was low in females than males which were statistically significant. Total cholesterol was significantly higher in female CAD patients than male CAD patients $(p=0.03)$. Both atherogenic (LDL) and protective (HDL) cholesterol were more in females than males with normal coronaries, but this difference was seen in patients with CAD in both sexes.

543 females and 551 males had normal coronaries. Overall prevalence of normal coronaries is more in females $(p=0.00)$. Prevalence of diabetes showed a tendency more in females with normal coronaries than with males $(p=0.063)$. Overall prevalence of risk factors in patients with normal coronaries is more in females compared to males ( $p=0.03) .782(48.87 \%)$ males and 301(55.94\%) females with CAD had diabetes. Prevalence of HTN $(p=0.00)$ and DM $(p=0.005)$ is more in
\end{abstract}

Article received on 25 March2016, published on 30 April 2016.

Deepti Galli ${ }^{1}$, Ravi Kiran ${ }^{1}$, Bharathi Vanapathi ${ }^{2}$

${ }^{1}$ DM 1 year student, Department of Cardiology, Nizam's Institute of Medical Sciences, India.

${ }^{2}$ Assistant Professor, Department of Cardiology

Corresponding author: Deepti Gali

Email: drdeepthicardiology@gmail.com females with CAD than males ( $p=0.00)$. Both DM EHTN was present in 647 (40.43\%) of males and 273 (50.74\%) of females, which is also is statistically significant.

Conclusion

Overall prevalence of normal coronaries is significantly more in females than males $(p=0.00)$. Incidence of ACS is significantly more in males whereas CSA incidence more in females. Even though statistically significant more prevalence HTN in females, there was no statistical significant difference in prevalence of DM $\mathcal{E} H T N$ in between both sexes in patients with normal coronaries. But there was significantly high prevalence of DM $\mathcal{E} H T N$ in females with CAD compared to males.

KEY WORDS: Coronary Angiogram, Gender differences.

\section{INTRODUCTION}

Coronary artery disease (CAD) is a leading cause of death of women and men worldwide [1,2]. A conservative estimate indicates that there could be 30 million CAD patients in India of which 14 million are in urban and 16 million in rural area. If the current trend continues by the year 2020, the burden of atherothrombotic CAD in India will surpass other regions of the world. [3]. Factors of risk for the premature CAD in Indian subjects could be multiple, ranging from social, economic, psychological, lifestyle (sedentary lifestyle, improper diet, smoking) and biological (abnormal lipids, hypertension, diabetes, and obesity). Genetic factors such as mutations at specific chromosomal locations and single nucleotide polymorphisms have also been implicated [4,5].

Risk factors for coronary artery disease are classified into modifiable and non modifiable. Non modifiable are age, family history and gender. Modifiable risk factors are high blood pressure, dyslipidemia, obesity, tobacco use, lack of physical activity, diabetes, psychosocial stress, low fruit and vegetables intake, heavy alcohol intake. Hypertension is more prevalent in women, particularly older women [6]. Meta-analysis of 37 prospective cohort studies showed the presence of diabetes is a relatively greater risk factor for CAD in women versus men, increasing a woman's risk of CAD 
by 3- to 7-fold, with only a 2- to 3-fold increase in diabetic men. Furthermore, women with diabetes have a greater than 3-fold increase in CAD risk than nondiabetic women do [7]. Gestational diabetes increases the risk for future diabetes [8], thereby increasing the risk for future CAD. So, we wanted to see the effect of DM on gender in Asian Indians referred for coronary angiogram (CAG).

\section{MATERIALS \& METHODS}

This study is a prospective observational study from the Nizam's institute of medical sciences (NIMS), which is a tertiary care level hospital and teaching institute. The study was conducted during January 2013 - December 2015. Two main risk factors diabetes \& hypertension was studied in the population of patients referred for CAG. In this cohort even all types of angina like Acute coronary syndrome (ACS), chronic stable angina (CSA), post MI(Myocardial infarction) angina, MI and chest pain with high pre-CAG probability of CAD were included. Patient with Valvular heart disease and congenital heart disease patients were excluded from study. Hypertension was defined using WHO criteriapressure exceeding a systolic value of $140 \mathrm{mmHg}$ and/or a diastolic of $90 \mathrm{mmHg}$. Patients on antidiabetic drugs or having a fasting blood glucose level $\geq 7.0 \mathrm{mmol} / \mathrm{L}$ were considered to be diabetic. We had compared the prevalence of diabetes, hypertension \& combination of these two risk factors, in males vs females. And we also compared the risk factors in patients with CAD \& normal coronaries.

\section{RESULTS}

During 24 month period, 3820 patients underwent CAG, but in 3232 cases the data is complete to analyse. Out of them 1081 females and 2151 males were there. 543 females and 551 males had normal coronaries. The details demographic and clinical presentations were given Table 1. $19.1 \%$ of male patients presented with ACS and $91.6 \%$ female patients were presented with CSA. LV dysfunction was present more in male patients than female patients $(31.3 \%$ vs $18.8 \%)$. Even when we sub classify the LV dysfunction into mild (41 to $49 \%$ ), moderate $(40$ to $31 \%)$ and severe $(<30 \%)$ depending on global EF, all severities of LV dysfunctions were more frequent in males than females.
In Table 2 gives the details of lab parameters. Serum Creatinine (SC) and Hemoglobin (HB) is low in females than males which are statistically significant (Fig 1). Total cholesterol was significantly higher in female CAD patients than male CAD patients $(p=0.03)$. Both atherogenic (LDL) and protective (HDL) cholesterol were more in females than males with normal coronaries, but this difference was seen in patients with CAD in both sexes (Fig 2).

Table 1: Demographic and clinical presentation of the study cohort.

\begin{tabular}{|l|l|l|l|}
\hline Parameters & Females & Males & p value \\
\hline Number & 1081 & 2152 & \\
\hline Age & $53.1 \pm 10.7$ & $53.2 \pm 11.8$ & 0.89 \\
\hline Height(cms) & $151.8 \pm 8.1$ & $159.6 \pm 11.4$ & 0.00 \\
\hline Weight(kgs) & $59.2 \pm 10.98$ & $62.7 \pm 10.8$ & 0.00 \\
\hline $\begin{array}{l}\text { Type of CAD } \\
\text { ACS }\end{array}$ & $\begin{array}{l}90(8.3 \%) \\
\text { CSA }\end{array}$ & $412(19.1 \%)$ & 0.00 \\
\hline $\begin{array}{l}\text { LV Dys.(yes) } \\
\text { Severity of LVD } \\
\text { Severe }\end{array}$ & $203(18.8 \%)$ & $673(31.3 \%)$ & 0.00 \\
$\begin{array}{l}\text { Moderate } \\
\text { Mild }\end{array}$ & $\begin{array}{l}74(6.8 \%) \\
57(5.3 \%)\end{array}$ & $224(10.4 \%)$ & 0.00 \\
\hline
\end{tabular}

Figure 1: Demonstration of less HB and Serum creatinine levels in female patients with normal coronaries.

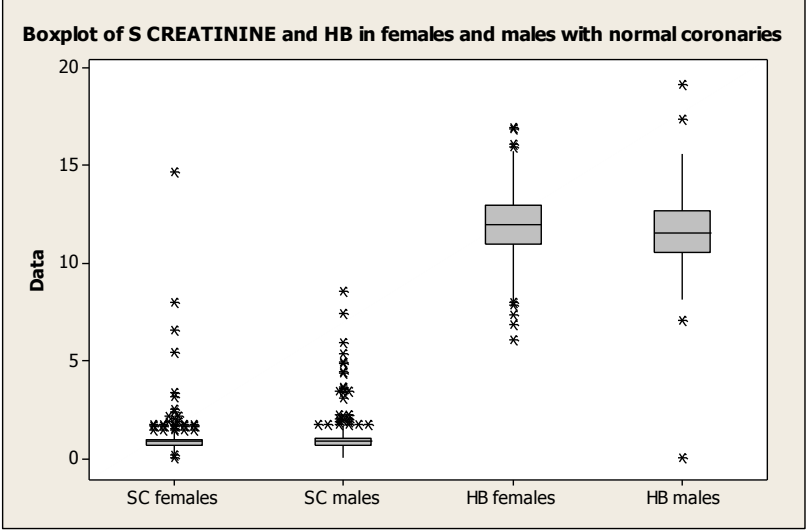


Table 2: Details of lab parameters

\begin{tabular}{|l|l|l|l|l|l|l|}
\hline Parameters & $\begin{array}{l}\text { Females with } \\
\text { NC }\end{array}$ & $\begin{array}{l}\text { Males with } \\
\text { NC }\end{array}$ & p value & $\begin{array}{l}\text { Females with } \\
\text { CAD }\end{array}$ & $\begin{array}{l}\text { Males with } \\
\text { CAD value }\end{array}$ & p \\
\hline Blood urea & $26.5 \pm 16.6$ & $28.5 \pm 18.5$ & 0.07 & $30.9 \pm 17.3$ & $30.7 \pm 18.1$ & 0.88 \\
\hline S. Cre & $0.96 \pm 0.8$ & $1.3 \pm 1.4$ & 0.00 & $1.1 \pm 0.8$ & $1.3 \pm 1.2$ & 0.00 \\
\hline RBS & $133.5 \pm 69.1$ & $125.6 \pm 63.6$ & 0.17 & $157.1 \pm 79.8$ & $152.9 \pm 76.3$ & 0.45 \\
\hline HB & $11.9 \pm 1.6$ & $13.3 \pm 2.0$ & 0.00 & $11.6 \pm 1.6$ & $13.2 \pm 2.0$ & 0.00 \\
\hline Cholesterol & $175.4 \pm 46.7$ & $160.2 \pm 43.4$ & 0.14 & $170.9 \pm 53.9$ & $151.9 \pm 47.8$ & 0.03 \\
\hline HDL & $47.3 \pm 11.7$ & $40.0 \pm 10.7$ & 0.01 & $41.5 \pm 11.2$ & $39.6 \pm 13.8$ & 0.35 \\
\hline LDL & $102.1 \pm 39.6$ & $79.6 \pm 32.7$ & 0.02 & $86.3 \pm 40.4$ & $94.5 \pm 111.8$ & 0.49 \\
\hline
\end{tabular}

Out of 2151 males, 551(25.6\%) males had normal coronaries, $1600(74.38 \%)$ males had CAD in CAG. Of 1081 females, $543(50.23 \%)$ females had normal coronaries and 538(49.76\%) had CAD. Overall prevalence of normal coronaries is significantly more in females than males $(\mathrm{p}=0.00)$.

Figure 2: LDL and HDL cholesterol levels in patients with normal coronaries

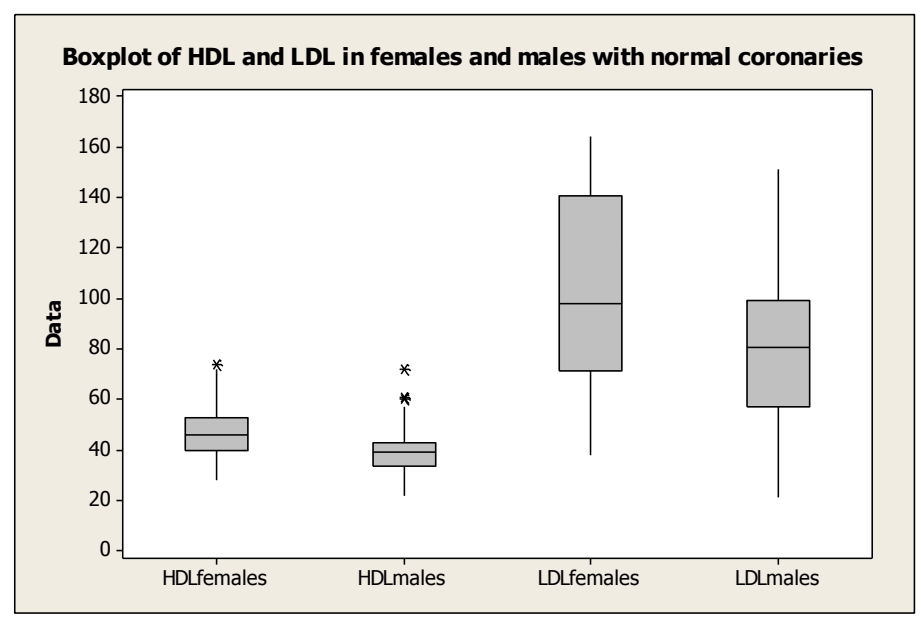

Prevalence of HTN \& DM in all patients presented for CAG was studied. There was more percentage of female patients had hypertension which was statistically significant, but there was in difference in DM prevalence (Table 3).
Table 3: DM and HTN prevalence in all patients referred for CAG.

\begin{tabular}{|l|l|l|l|}
\hline Parameter & Females & Males & p value \\
\hline HTN & $706(65.3 \%)$ & $1312(60.9 \%)$ & 0.02 \\
\hline DM & $496(45.9 \%)$ & $950(44.1 \%)$ & 0.35 \\
\hline
\end{tabular}

Prevalence of DM \& HTN in patients with normal coronaries is given in Table 4 and In CAD in Table 5. There is no statistical significant difference in prevalence of DM \&HTN in males \& females with normal coronaries (Fig 3).

Table 4: Risk factor distribution in patients with normal coronaries

\begin{tabular}{|l|l|l|l|}
\hline Parameter & Females & Males & $\mathrm{p}$ value \\
\hline HTN & $307(56.5 \%)$ & $307(55.7 \%)$ & 0.808 \\
\hline DM & $195(35.9 \%)$ & $168(30.5 \%)$ & 0.063 \\
\hline HTN+DM & $150(27.6 \%)$ & $138(25.04 \%)$ & 0.337 \\
\hline RF present & $394(72.6 \%)$ & $355(64.4 \%)$ & 0.032 \\
\hline
\end{tabular}

Table 5: Risk factor profile in patients with CAD.

\begin{tabular}{|l|l|l|l|}
\hline Parameter & Females & Males & p value \\
\hline Hypertension & $399(74.1 \%)$ & $1005(62.8 \%)$ & 0.000 \\
\hline DM & $301(55.9 \%)$ & $782(48.9 \%)$ & 0.005 \\
\hline HTN+DM & $273(50.7 \%)$ & $647(43.4 \%)$ & 0.000 \\
\hline RF present & $432(80.3 \%)$ & $1277(79.8 \%)$ & 0.852 \\
\hline
\end{tabular}


There is significantly high prevalence of DM \&HTN in females with CAD compared to males (Fig 4).

Fig 3: Showing significant more prevalence of DM in females with Normal coronaries.

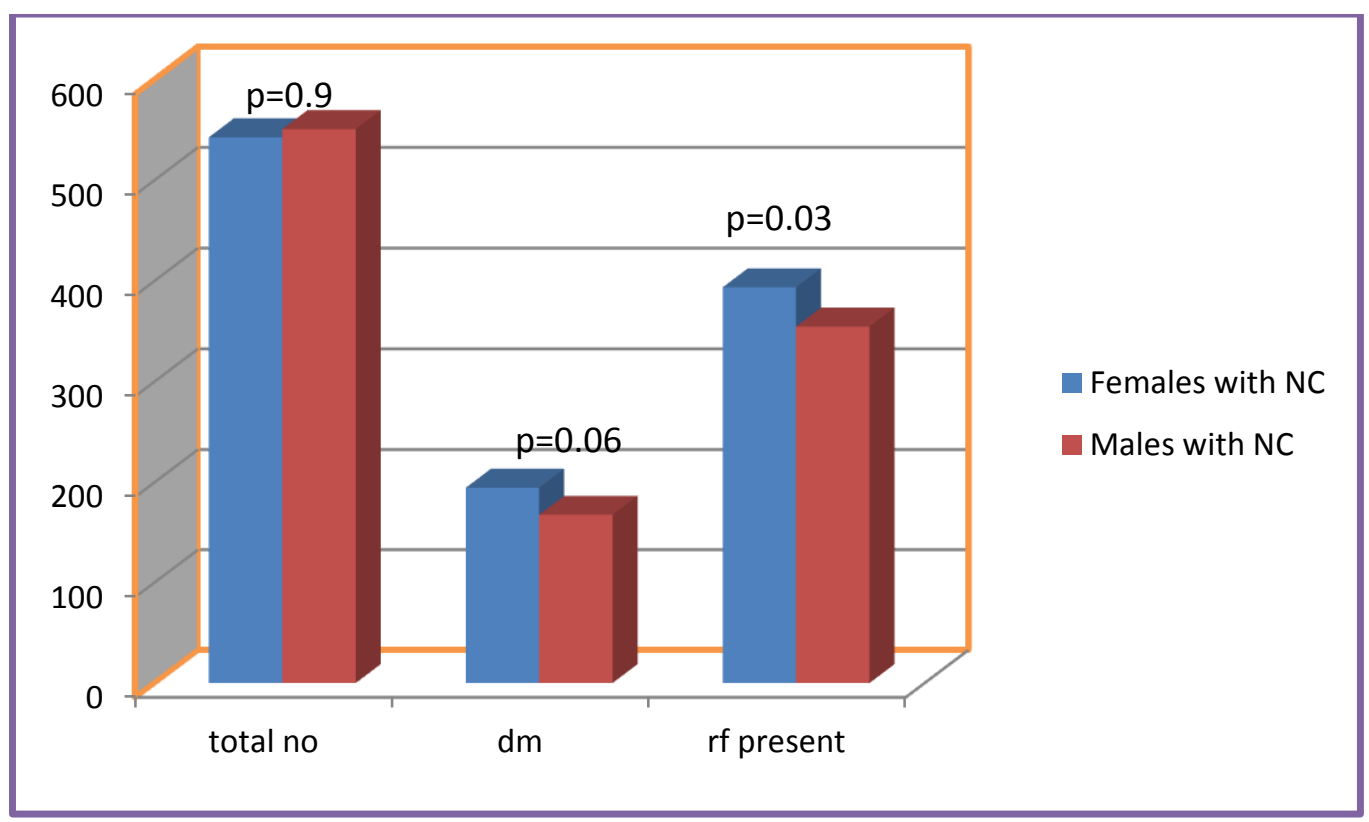

Figure 4: Comparison of DM and HTN in Patients with

CAD in both sexes.



\section{CONCLUSION:}

It is known that when female patients present with chest pain, they are more likely to normal coronary arteries than in males with chest pain. Yusuf et al in Inter heart study $[8\}$ showed prevalence of normal coronaries is significantly more in females than males. Female CAD patients present with different set of symptoms and their symptoms do not have same relationship to the extent and severity of coronary artery disease as their male counterparts. Females tend to present later in age by about 10 years and are likely to have less severe coronary artery disease with worse symptoms. In contrast to the linear increase in CAD in men as they age, there is a more exponential increase in CAD in women after the age of $60[9,10]$. Whereas We not have studied the sub group analysis of age group above or below the 60 years in this study cohort. 
In INTER HEART study, Under-referral of this subset of high risk female patients for cardiac catheterization compared to their increased risk has been demonstrated over a follow up period of 1 year. It has also been shown in various studies that female CAD patients have an adverse risk factor profile for the prevalence of diabetes mellitus, hypertension and hypercholesterolemia. We have clearly demonstrated the same finding in our current study also. In this normal coronaries were more in females than in males $(p=0.00)$. Similarly, HTN was significantly higher in prevalence in our female patients undergoing CAG $(\mathrm{p}=0.02)$. In subgroup analysis of patients undergone CAG, there was significantly high prevalence of DM \& HTN in females with CAD compared to males.

The burden of CAD is high among women. However, it appears that the pathophysiology of CAD varies between women and men. On cardiovascular computed tomography, women have been shown to have smaller coronary artery diameters than men do [10]. Women are less likely than men are to have obstructive CAD at the time of coronary angiography [11,12]. Despite the lack of obstructive CAD visualized on cardiac catheterization at the time of acute coronary syndrome (ACS), the prognosis of these women is not benign. Over one-half of symptomatic women without obstructive CAD continue to have signs and symptoms of ischemia and to undergo repeat hospitalization and coronary angiography $[12,13]$.

Latest PROMISE trail by Hemal K et [14] also showed some of the similar features of our study like women were older (62.4 years of age vs. 59.0 years of age) and were more likely to be hypertensive $(66.6 \%$ vs. $63.2 \%)$, dyslipidemic ( $68.9 \%$ vs. $66.3 \%)$, and to have a family history of premature CAD (34.6\% vs. 29.3) (all p values $<0.005)$. Women were less likely to smoke $(45.6 \%$ vs. $57.0 \%$; $\mathrm{p}<0.001)$, although their prevalence of diabetes was similar to that in men $(21.8 \%$ vs. $21.0 \%$; $=0.30)$. Chest pain was the primary symptom in $73.2 \%$ of women versus $72.3 \%$ of men $(p=0.30)$, and was characterized as "crushing/ pressure/ squeezing/ tightness" in $52.5 \%$ of women versus $46.2 \%$ of men $(\mathrm{p}<$ 0.001). Compared with men, all risk scores characterized women as being at lower risk, and providers were more likely to characterize women as having a low $(<30 \%)$ pre-test probability of CAD $(40.7 \%$ vs. $34.1 \%$; p < 0.001$)$. Compared with men, women were more often referred to imaging tests (adjusted odds ratio: 1.21; 95\% confidence interval: 1.01 to 1.44$)$ than non-imaging tests. Women were less likely to have a positive test $(9.7 \%$ vs. $15.1 \% ; \mathrm{p}<0.001)$. Although univariate predictors of test positivity were similar, in multivariable models, age, body mass index, and Framingham risk score were predictive of a positive test in women, whereas Framingham and Diamond and Forrester risk scores were predictive in men.

\section{LIMITATIONS:}

Major limitation of our study is we studied in the patient group referred for CAG, which are more likely to have more risk factors. So, this data is not applicable where to choose or not to choose the invasive assessment of CAD, when patients are referred for CAG in the general population. Another limitation was we had taken presence or absence of DM \& HTN but not the duration of these risk factors which influences the occurrence of CAD. We had taken presence or absence of CAD but not the severity of CAD.

\section{REFERENCES:}

1) Rajeeva Rivikath Pieris, Hilal Ali Al-Sabti, Qasim Saleh Abdullah Al-Abri and Syed Gauhar Alam Rizvi. Prevalence pattern of risk factors for coronary artery disease among patients presenting for coronary artery bypass grafting in Oman. Oman Medical Journal. 2014; 29 (3): 203-207.

2) Kou-Gi Shyu, Chiung-Jen $\mathrm{Wu}$, Guang-Yuan Mar, Charles Jia-Yin Hou, Ai-Hsien Li, Ming-Shien Wen. Clinical characteristics, management and in-Hospital outcomes of patients with acute coronary syndrome observations from the Taiwan ACS full spectrum registry. Acta Cardiol Sin 2011; 27:135-144

3) Enas A Enas, Vibhuti Singh, YP Munjal, Suman Bhandari, Ram Dev Yadave, SC Manchanda. Reducing the burden of coronary artery disease in India: Challenges and Opportunities. Indian Heart J 2008; 60: 161-175.

4) Raja Babu Panwar, Rajeev Gupta, Bal Kishan Gupta, Sadiq Raja, Jaishree Vaishnav, Meenakshi Khatri \& Aachu Agrawal. Atherothrombotic risk factors \& 
premature coronary heart disease in India: A casecontrol study. Indian J Med Res, 2011; 134: 26-32.

5) Prashanth Kulkarni. Family history of coronary artery disease as an Additional Risk Factor Associated with Coronary Artery Disease: A Descriptive Observational Study. Journal of Clinical Trials in Cardiology. 2015; 2(1): 1-3.

6) Centers for Disease Control and Prevention. Health, United States, 2009: With Special Feature on Medical Technology [pdf]. 2010. Available from: http://www.cdc.gov/nchs/data/hus/hus11.pdf.Access ed April 12, 2013.

7) Huxley R, Barzi F, Woodward M. Excess risk of fatal coronary heart disease associated with diabetes in men and women: meta-analysis of 37 prospective cohort studies. BMJ 2006;332:73-8.

8) Yusuf S1, Hawken S, Ounpuu S, Dans T, Avezum A, Lanas F, McQueen M, Budaj A, Pais P, Varigos J, Lisheng L; INTERHEART Study Investigators. Effect of potentially modifiable risk factors associated with myocardial infarction in 52 countries (the INTERHEART study): case-control study. Lancet. 2004 Sep 11-17;364(9438):937-52.

9) Shaw LJ, Merz CN, Pepine CJ, et al, for the WISE Investigators. The economic burden of angina in women with suspected ischemic heart disease: results from the National Institutes of Health National Heart, Lung, and Blood Instituteesponsored Women's Ischemia Syndrome Evaluation. Circulation 2006;114:894-904

10) Thom TJ, Kannel WB, Silbershatz H, D'Agostino RB Sr. Cardiovascular diseases in the United States and prevention approaches. In:Fuster V, Alexander RW, O'Rourke RA, Roberts R, Spencer BK III,Weller JJ, eds. Hurst's the Heart. 10 edition. New York, NY: McGrawHill; 2001. 3-1.

11) Ratner RE. Prevention of type 2 diabetes in women with previous gestational diabetes. Diabetes Care 2007; 30(Suppl 2):S242-5.
12) Sharaf BL, Pepine CJ, Kerensky RA, et al, for the WISE Study Group. Detailed angiographic analysis of women with suspected ischemic chest pain (pilot phase data from the NHLBI-sponsored Women's Ischemia Syndrome Evaluation [WISE] Study Angiographic Core Laboratory). Am J Cardiol 2001;87: 937-41, A3.

13) Kennedy JW, Kilip T, Fisher LD, Alderman EL, Gillespie MJ, Mock MB. The clinical spectrum of coronary artery disease and its surgical and medical management, 1974e1979. The Coronary Artery Surgery study. Circulation 1982;66: III16-23.

14) Johnson BD, Shaw LJ, Pepine CJ, et al. Persistent chest pain predicts cardiovascular events in women without obstructive coronary artery disease: results from the NIH-NHLBIesponsored Women's Ischaemia Syndrome Evaluation (WISE) study. Eur Heart J 2006;27: 1408-15. 